\title{
A YOUNG MALE WITH FEMALE REPRODUCTIVE SYSTEM
}

\author{
MD ROBED AMIN ${ }^{1}$, AMIT DAS $^{2}$, MD MURAD HOSSAIN ${ }^{3}$, MD FERDOUS ZAMAN $^{4}$, AHMEDUL KABIR ${ }^{1}$, \\ HAFIZUL ISLAM ${ }^{1}$, M A KASHEM KHONDOKAR ${ }^{5}$
}

\begin{abstract}
Patients with disorders of sexual differentiation possess discordance among sex chromosomes, gonads, sex hormones, and phenotypic sex. The most common of the phenotypic sex disorder is due to defect in enzyme for synthesis of steroids in adrenal cortex. A spectrum of phenotypes is observed in these cases. Here we present a case with features of female reproductive system in his body with male phenotype. As there was no trace of male sexual organs in all investigations but physical habitus was exclusively male with abundance of hair distribution the case was diagnosed as disorder of sex development (DSD) - female pseodoharmaphroditism.
\end{abstract}

Keywords: Sex Differentiation Disorder, Disorders of Sexual Development, Pseudohermaphroditism

\section{Introduction}

The chromosomal sex disorder is governed by their chromosomal status and internal reproductive organ is formed by their sex chromosomal direction ${ }^{1,2}$. The gonadal sex disorder can have both testes and ovary run simultaneously and differentiation depends on their dominant part activity. The phenotypic sex disorder is due to differentiation of their phenotypic expression on firm chromosomal status. The most common of the phenotypic sex disorder is due to defect in enzyme for synthesis of steroids in adrenal cortex. ${ }^{3}$ The most frequent is steroid 21-hydroxylase deficiency, accounting for more than 90 percent of cases. ${ }^{4} \mathrm{~A}$ spectrum of phenotypes is observed in these cases. In patients with disorders of sexual differentiation, there is discordance among sex chromosomes, gonads, sex hormones, and phenotypic sex (internal reproductive structure and external genital appearance). These conditions include true hermaphroditism (in which both ovarian and testicular tissue are present in the same or opposite gonads); 46,XX individuals with congenital adrenal hyperplasia, particularly those born with marked or complete masculinization of the external genitalia; 46,XY individuals with partial androgen insensitivity; 46,XY individuals with 5-\{alpha\}-reductase deficiency;
46,XY individuals with a very small but normally formed penis (micropenis) or malformed penis (microphallus); and 46,XY individuals with cloacal exstrophy.

\section{Case report}

Mr. O. K, 28 yrs old bachelor with permanent residence in Khulna, admitted in Dhaka Medical College Hospital (DMCH) with the history of intermittent abdominal pain for 12 years and gradual distension of abdomen for 10 years. The pain was dull aching in nature, started from back, radiated to front of the lower abdomen and upper part of the back, moderate in severity, associated with vomiting, lasted for 3 to 4 days in almost every month, aggravated on sitting position and relieved on lying posture and /or taking NSAIDs like tramadol hydrochloride. Due to recurrence of similar episodes of pain, he took advice from different physician without any benefit. He later took an advice from a surgeon who found a mass in abdomen and advised for removal of the mass. After an operative procedure to remove the mass he noticed a feeling of abdominal distension and a mass in the left side of the middle of abdomen. The mass was gradually increasing in size. He had no bladder or bowel disturbance. His

1. Assistant Professor, Department of Medicine, Dhaka Medical College

2. Honorary Medical Officer, Department of Medicine, Dhaka Medical College Hospital

3. Assistant Registrar, Department of Medicine , Dhaka Medical College Hospital

4. Registrar, Department of Medicine, Dhaka Medical College Hospital

5. Professor, Department of Medicine, Shaheed Suhrawardy Medical College

Correspondence: Dr. Md Robed Amin, Assistant Professor, Department of Medicine, Dhaka Medical College. E-mail: robedamin@yahoo.com 
vomiting was on and off and the pain, although lesser than before, bothered his life style. On query, he told that although he had a robust body in his small frame, he had poorly developed external genitalia and he never felt his testes. He also had a history of whitish discharge per urethra for which he was treated conservatively. Interestingly his discharge per urethra was on a regular monthly basis. There were no other symptoms like cough, chest pain, breathlessness, headache, anorexia or ENT related ailments during any period of his illness. His sexual urge was normal for his age but he refused to get married as his testes were absent and become a religious person.

On general examination of the patient, he was grossly hyperpigmented with long beard, having frontal baldness, short stature with small body frame (height-51 inches, weight-33 kg, waist-28 inches, chest-30 inches, arm circumference 7 inches, upper limb length 18 inches) and short $4^{\text {th }}$ toe in both foot. During admission his vitals were pulse- $88 / \mathrm{min}, \mathrm{BP}$ -

$85 / 70 \mathrm{~mm}$ of $\mathrm{Hg}$, temerature- $36.4^{\circ} \mathrm{C}$, respiratory rate $-16 / \mathrm{min}$. On systemic examination of gastrointestinal tract and genitourinary system, there was a soft, mild tender, irregular, ill-defined, movable mass in the left hypochondriac and left lumber region, measuring $20 \mathrm{x} 15 \mathrm{~cm}$, extending to epigastrium and umbilicus, having a cleft between epigastrium and umbilicus. Another mass was felt in the right upper abdomen measuring about $10 \times 9 \mathrm{~cm}$ with same characteristics. There was no ascites. Testes couldn't be felt, no definite scrotal sac but a tag of skin was observed at external genitalia. There was phallus like clitoris which was extremely prominent mimicking penis. Other systems revealed no abnormality.

On investigation, complete blood count was within normal limit, s. creatinine -96 micromol/L, RBS -3.8 $\mathrm{mmol} / \mathrm{L}$, SGPT-25 U/L, urine R/M/E- albumin (+), hormonal assay- testosterone $39.3 \mathrm{nmol} / \mathrm{l}, \mathrm{LH}-0.73$ $\mathrm{miu} / \mathrm{ml}$, FSH-0.95 miu/ml, oestrogen $79.73 \mathrm{pg} / \mathrm{ml}$, progesterone $41 \mathrm{ng} / \mathrm{ml}, 24 \mathrm{hrs}$ urinary cortisol $429.04 \mathrm{nmol} /$ day, $17(\mathrm{OH})$ progesterone $4.5 \mathrm{ng} / \mathrm{ml}$. Chest X-ray showed left sided basal pleural effusion, abdominal X-ray showed abdominal mass and/or ascites, upper GIT endoscopy revealed gastritis, ultrasonogram of whole abdomen including inguinoscrotal region showed prostate was not visualized, uterus was seen instead of prostate, ovaries were also visualized and normal in size, testes could not be isolated from the ovarian structure in the pelvic region (Figure 1). There was crossed ectopia of the slightly smaller left kidney in the right pelvic region with malrotation. There was huge bowel gases with distended bowel loops mainly in left lower abdomen, pelvic region, extended up to right mid abdomen and left hypochondriac region suggestive of volvulous of sigmoid colon. FNAC of abdominal mass was suggestive of lipoma, CT scan of abdomen revealed possibilities of retroperitoneal lipomas or bilateral adrenal myolipomas. Other findings were consistent with ultrasonographic findings. Intravenous urography showed ectopic left kidney. Morphological outline of left kidney was not discernible. Excretory functions of both the kidneys were normal. Cystogram was of normal appearance. No post voidal residue was present.

His examination and investigations revealed features of female reproductive system in his body with male phenotype. As there was no trace of male sexual organs in all investigations but physical habitus was exclusively male with abundance of hair distribution, the case was diagnosed as disorder of sex development (DSD) - female pseodoharmaphroditism.

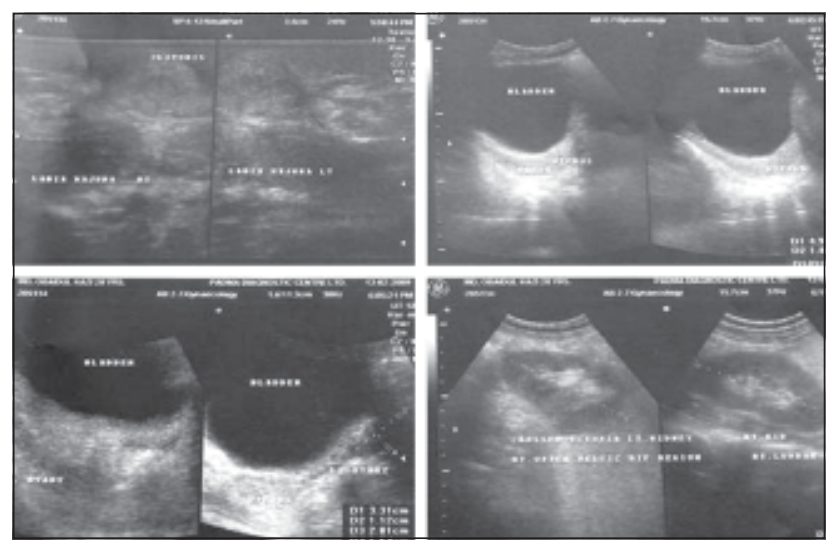

Fig.-1: Ultasonogram of abdomen showing ovary and uterus.

\section{Discussion}

Sex development begins in utero but continues into young adulthood with the achievement of sexual maturity and reproductive capability. The early stages of sex development can be divided into three major components: chromosomal sex, gonadal sex (sex determination), and phenotypic sex (sex 
differentiation). Abnormalities at each of these stages can result in disorders of sex development (DSD). ${ }^{1}$ DSD can manifest later in life due to subtler forms of gonadal dysfunction [e.g. non-classical congenital adrenal hyperplasia (DSD)] and are often diagnosed by internists. ${ }^{2}$ The chromosomal sex disorder is governed by their chromosomal status and internal reproductive organ is formed by their sex chromosomal direction. The gonadal sex disorder can have both testes and ovary (ovotestes) run simultaneously and differentiation depends on their dominant part activity. The phenotypic sex disorder is due to differentiation of their phenotypic expression on firm chromosomal status. The most common of the phenotypic sex disorder is due to defect in enzyme for synthesis of steroids in adrenal cortex. ${ }^{3}$ The most frequent is steroid 21-hydroxylase deficiency, accounting for more than 90 percent of cases. ${ }^{4}$ A spectrum of phenotypes is observed. A severe form with a concurrent defect in aldosterone biosynthesis (salt-wasting type) and a form with apparently normal aldosterone biosynthesis (simple virilizing type) are together termed classic 21hydroxylase deficiency. There is also a mild, nonclassic form that may be asymptomatic or associated with signs of postnatal androgen excess. ${ }^{5}$ The classical forms are more common in infant or childhood and severe form of disorder as the child are more prone to develop shock, electrolytes imbalance, high renin and low aldosterone. ${ }^{6}$ In our case the patient had unremarkable childhood and raised as girl with ambigous genitalia. Such girls have ambiguous genitalia: a large clitoris, rugated and partially fused labia majora, and a common urogenital sinus in place of a separate urethra and vagina. The uterus, fallopian tubes, and ovaries are normally formed, but there is no development of the wolffian duct. In patients who either are not treated or are inadequately treated, long-term exposure to high levels of sex hormones promotes rapid somatic growth (predominantly an androgen effect) and advanced skeletal age, which leads to premature epiphyseal fusion (predominantly an effect of extragonadal aromatization of androgens to estrogens). Pubic and axillary hair may develop early. In our patient there was also rapid growth and good masculine body developed in adolescent age and axillary and pubic hair grows very fast. The family raised the child as boy during this period and psychosocially adapted and accepted it without taking any physician advice. Linear growth is affected by congenital adrenal hyperplasia. Both undertreatment and overtreatment put patients at risk for short stature, the former causing premature epiphyseal closure induced by high levels of sex steroids and the latter resulting in glucocorticoid-induced inhibition of the growth axis. ${ }^{7,8,9}$ In our case he is short statured with frontal baldness and huge beard (hirsutism) indicating androgen excess in body. In girls with any form of 21-hydroxylase deficiency, signs of reproductive problems, such as oligomenorrhea or amenorrhea, may develop in adolescence ${ }^{10,11}$. The issue of fertility is inextricably related to psychosocial adjustment. In our patient during adolescence, she experienced monthly discharge through prominent phallus but which gradually decreased as age advanced. Although she was heterosexual and sexual identity was genuinely female, family brought her up as male and her male name socially was accepted due to her body build and hirsutism. Hirsutism is the single most common symptom at presentation in approximately 60 percent of symptomatic women, followed by oligomenorrhea (54 percent) and acne (33 percent). ${ }^{12}$ Thus, nonclassic 21-hydroxylase deficiency and polycystic ovarian syndrome may present in similar ways. Our patient came to attention to physician only when he started to feel mass and pain in abdomen. Laparotomy and excision of mass revealed fatty tissue as adrenal mass is basically fat containing tissue. During this time also the FNAC of abdominal mass revealed features of lipoma indicating proliferation of adrenal tissue. Classic 21-hydroxylase deficiency is characterized by markedly elevated serum levels of 17-hydroxyprogesterone. To improve accuracy, some screening programs have set reference levels for serum 17-hydroxyprogesterone in infants that are based on weight and gestational ages. whereas others have emphasized concurrent measurement of serum cortisol.13,14,15 Measurement of 17 hydroxyprogesterone by fluoro-immunoassay or tandem mass spectrometry may improve both the sensitivity and the specificity of screening. ${ }^{16,17}$ In our patient 17-hydroxyproesterone although raised was not at that level of classical form of CAH. The patient family declined any further investigation as they were concerned that her feminine identity would be established. Other hormones whose levels are usually elevated in patients with 21-hydroxylase 
deficiency include progesterone, androstenedione, and to a lesser extent, testosterone was found in our patient. Patients with classic 21-hydroxylase deficiency require long-term glucocorticoid treatment to inhibit excessive secretion of corticotropinreleasing hormone and corticotropin by the hypothalamus and pituitary, respectively, and to reduce elevated levels of adrenal sex steroids. The preferred drug is hydrocortisone (i.e. cortisol itself) inmaintenance doses of 10 to $20 \mathrm{mg}$ per square meter of body-surface area per day in three divided doses. The therapeutic goal is to use the lowest dose of glucocorticoid that adequately suppresses adrenal androgens and maintains normal growth and weight gain. ${ }^{18}$ The non classical form do not need aldosterone as there is no deficit of this hormone. The reconstruction surgery for phallus enlargement could not be done due to noncompliance on the part of family members. Although medical management of different steroids is still the hallmark of treatment in this unfortunate disease, the steroid side effects get many physicians concerned. The adrenalectomy by surgeons although seems promising, but crisis may ensue after operation. Finally, a genetically wellcharacterized disease of 21-Hydroxylase deficiency might eventually be amenable to gene therapy. ${ }^{19}$ The implementation of such an approach for this as well as other metabolic diseases awaits a safe, effective, and stable tissue-specific delivery system.

\section{References}

1. Disorders of Sex Development. In: Fauci AS, Kasper DL, Longo DL, et al, eds Harrison's Internal Medicine. New York:McGraw-Hill Companies, 2008:3886-3887.

2. Ogilvie $\mathrm{CM}$ et al. Congenital adrenal hyperplasia in adults: A review of medical, surgical and psychological issues. Clin Endocrinol 2006;64:2.

3. White PC, New MI, Dupont B. Congenital adrenal hyperplasia. N Engl J Med 1987;316:1519-24.

4. Speiser PW, Dupont B, Rubinstein P, et al. High frequencyof nonclassical steroid 21-hydroxylase deficiency. Am J Hum Genet 1985;37:650-67.

5. White PC, Speiser PW. Congenital adrenal hyperplasia due to 21-hydroxylase deficiency. Endocr Rev 2000;21:245-91.

6. Fukushige J, Simomura K, Ueda K. Influence of upright activity on plasma renin activity and aldosterone concentration in children. Eur J Pediatr 1994;153:284-6.

7. Eugster EA, Dimeglio LA, Wright JC, et al. Height outcome in congenital adrenal hyperplasia caused by 21-hydroxylase deficiency: a meta-analysis. $\mathrm{J}$ Pediatr 2001;138:26-32.
8. Jaaskelainen J, Voutilainen R. Growth of patients with 21-hydroxylase deficiency: an analysis of the factors influencing adult height. Pediatr Res 1997;41:30-3.

9. Yu AC, Grant DB. Adult height in women with earlytreated congenital adrenal hyperplasia (21hydroxylase type): relation to body mass index in earlier childhood. Acta Paediatr 1995;84:899-903.

10. Barnes RB, Rosenfield RL, Ehrmann DA, et al. Ovarian hyperandrogynism as a result of congenital adrenal virilizing disorders: evidence for perinatal masculinization of neuroendocrine function in women. J Clin Endocrinol Metab 1994;79:1328-33.

11. Deneux C, Tardy V, Dib A, et al. Phenotype- genotype correlation in 56 women with nonclassical congenital adrenal hyperplasia due to 21-hydroxylase deficiency. J Clin Endocrinol Metab 2001;86:20713.

12. Moran C, Azziz R, Carmina E, et al. 21- Hydroxylasedeficient nonclassical adrenal hyperplasia is a progressive disorder: a multicenter study. Am J Obstet Gynecol 2000; 183:1468-74.

13. Allen DB, Hoffman GL, Fitzpatrick P, et al. Improved precision of newborn screening for congenital adrenal hyperplasia using weightadjusted criteria for 17-hydroxyprogesterone levels. J Pediatr 1997;130:128-33.

14. Gruneiro-Papendieck L, Prieto L, Chiesa A,et al. Neonatal screening program for congenital adrenal hyperplasia: adjustments to the recall protocol. Horm Res 2001;55:271-7.

15. Mikami A, Fukushi M, Oda H, et al. Newborn screening for congenital adrenal hyperplasia in Sapporo City: sixteen years experience. Southeast Asian J Trop Med Public Health 1999;30:Suppl 2:100-2.

16. Boudi A, Giton F, Galons H, et al. Development of a plasma 17alpha- hydroxyprogesterone time resolved-fluorescence immunoassay involving a new biotinylated tracer. Steroids 2000;65:103-8.

17. Minutti C, Magera MJ, Casetta BN, et al. Analysis of $17-\mathrm{OH}$ progesterone (17OHP) by tandem mass spectrometry (MS/MS) for the detection of congenital adrenal hyperplasia (CAH) in newborn blood spots. J Inherit Metab Dis 2001;24:Suppl 1:10.

18. Migeon CJ, Wisniewski AB. Congenital adrenal hyperplasia owing to 21-hydroxylase deficiency: growth, development, and therapeutic considerations. Endocrinol Metab Clin North Am 2001;30:193-206.

19. Tajima T, Okada T, Ma XM, et al. Restoration of adrenal steroidogenesis by adenovirus-mediated transfer of human cytochrome P450 21-hydroxylase into the adrenal gland of 21-hydroxylase-deficient mice. Gene Ther 1999;6:1898-903. 